\title{
FLEXIBLE BATTERIES
}

\section{Core-double shell}

Energy Environ. Sci. http://doi.org/cp5d (2018)

Flexible electronic devices require flexible power systems. Rechargeable batteries can be made flexible, but they suffer from low energy densities compared to rigid batteries. This is because active electrode materials often need to be grown on flexible substrates that carry weight but do not contribute much to the stored energy. Furthermore, the mass loading of active materials is often low, also limiting the energy density. Additional challenges are associated with mechanical stability of electrode materials on flexible substrates. Now, Zhao-Qing Liu, Yexiang Tong and colleagues in China have developed a flexible battery based on a carbon cloth coated with a porous carbon shell as well as a porous $\mathrm{NiCo}_{2} \mathrm{O}_{4}$ nanowire shell to tackle the aforementioned problems.

To fabricate the battery, the researchers grew a $\mathrm{NiCo}_{2} \mathrm{O}_{4}$ precursor onto a carbon cloth substrate, which was followed by a thermal reduction and subsequent etching. Aided by theoretical modelling, the researchers showed that the obtained core-double-shell structure displays a strong electronic interaction between the porous carbon and the $\mathrm{NiCo}_{2} \mathrm{O}_{4}$ nanowire, and that the interfacial interaction increases the electronic conductivity and facilitates Li-ion transport. In addition, compared to pristine carbon cloth, the carbon cloth with the porous carbon shell exhibits enhanced lithium-storage properties. The researchers constructed a full cell with this core-double-shell structured material as a high mass loading anode and paired it with a $\mathrm{LiNiCoMnO} \mathrm{O}_{2}$ cathode that was cast onto a similar carbon core-shell substrate. Based on the total weight of the cell, the resulting battery achieved an energy density of $314 \mathrm{Wh} \mathrm{kg}^{-1}$.

Changjun Zhang

Published online: 8 June 2018

https://doi.org/10.1038/s41560-018-0186-0 